\title{
Corrigendum: TNFR2 Is a Crucial Hub Controlling Mesenchymal Stem Cell Biological and Functional Properties
}

\author{
Ghada Beldi ${ }^{1 \dagger}$, Sheyda Bahiraii ${ }^{1,2 \dagger}$, Chloé Lezin ${ }^{1,3}$, Mahsa Nouri Barkestani ${ }^{1}$, \\ Mohamed Essameldin Abdelgawad ${ }^{1,3,4}$, Georges Uzan ${ }^{1,3 *}$ and Sina Naserian ${ }^{1,3,5 *}$ \\ ${ }^{1}$ INSERM UMR-S-MD 1197, Hôpital Paul Brousse, Villejuif, France, ${ }^{2}$ Department of Pharmacognosy, University of Vienna, \\ Vienna, Austria, ${ }^{3}$ Paris-Saclay University, Villejuif, France, ${ }^{4}$ Biochemistry Division, Chemistry Department, Faculty of Science, \\ Helwan University, Cairo, Egypt, ${ }^{5}$ CellMedEx, Saint-Maur-des-Fossés, France
}

Keywords: mesenchymal stem cells, immunoregulation, immune-checkpoint, tissue regeneration, angiogenesis, TNF $\alpha$-TNFR2 signaling pathway

\section{A Corrigendum on}

OPEN ACCESS

Edited and reviewed by: Songlin Wang,

Capital Medical University, China

${ }^{*}$ Correspondence:

Sina Naserian

sina.naserian@inserm.fr;

sina.naserian@cellmedex.com

Georges Uzan

georges.uzan@inserm.fr

tThese authors have contributed equally to this work

Specialty section:

This article was submitted to

Stem Cell Research

a section of the journal

Frontiers in Cell and Developmental

Biology

Received: 04 August 2021 Accepted: 06 August 2021

Published: 24 August 2021

Citation:

Beldi G, Bahiraii S, Lezin C, Nouri Barkestani M, Abdelgawad ME,

Uzan G and Naserian S (2021) Corrigendum: TNFR2 Is a Crucial Hub Controlling Mesenchymal Stem Cell Biological and Functional Properties. Front. Cell Dev. Biol. 9:753407. doi: 10.3389/fcell.2021.753407
TNFR2 Is a Crucial Hub Controlling Mesenchymal Stem Cell Biological and Functional Properties

by Beldi, G., Bahiraii, S., Lezin, C., Nouri Barkestani, M., Abdelgawad, M. E., Uzan, G., et al. (2020). Front. Cell Dev. Biol. 8:596831. doi: 10.3389/fcell.2020.596831

In the original article, there were two mistakes in the legend for Figure 5 and in Figure 7A.

Regarding the legend for Figure 5, we neglected to mention that the graphical images in Figure 5A were created with BioRender.com. The correct version appears as below.

Figure 5. The TNFR2 Expression by mesenchymal stem cells (MSCs) is associated with the induction of Tregs with more immunosuppressive effect. (A) T cells were freshly isolated and depleted from CD25 subpopulation in order to eliminate natural Tregs and highly activated T cells. $\mathrm{CD}^{+}{ }^{+} \mathrm{CD} 25^{-}$Tconvs (orange population) were then added to wild-type (WT) and TNFR2 knockout (KO)-MSCs in a fixed 1/10 MSC/T cell ratio. After $72 \mathrm{~h}, \mathrm{CD} 4{ }^{+} \mathrm{CD} 25^{+} \mathrm{Foxp} 3^{+}$induced regulatory $\mathrm{T}$ cells (iTregs) generated in those co-cultures (green population) were put in an mixed lymphocyte reaction (MLR) test with newly isolated and activated mouse $\mathrm{CFSE}^{+} \mathrm{CD}_{3}{ }^{+} \mathrm{CD} 25^{-}$ Tconvs in a fixed $1 / 5 \mathrm{iTreg} / \mathrm{Tconv}$ ratio. Then, the $\mathrm{CD} 4^{+}$and $\mathrm{CD} 8^{+}$proliferation capacity was measured by fluorescence-activated cell sorting (FACS). (B) Percentage of proliferation of CD4 ${ }^{+}$ and $\mathrm{CD}^{+}$Tconvs in the presence of MSC induced Foxp3 ${ }^{+}$Tregs. Control groups consist of unstimulated $\mathrm{T}$ cells alone as depicted by the white columns $(n=6)$, while the stimulated $\mathrm{T}$ cells alone are depicted by the black columns $(n=6)$. The blue columns represent the stimulated T cells co-cultured with iTregs derived from WT-MSCs $(n=6)$, and the red columns represent the stimulated T cells co-cultured with iTregs derived from TNFR2 KO-MSCs $(n=6)$. Results are collected from two independent experiments. The graphical images were created with BioRender.com.

Regarding Figure 7A, the representative images used for control groups i.e., MSCs WT and TNFR2 KO in DMEM medium were both from the MSC WT group. New representative images from proper control groups were added in the corrected version of Figure 7. The corrected Figure 7 appears below.

The authors apologize for these errors and state that this does not change the scientific conclusions of the article in any way. The original article has been updated. 
A

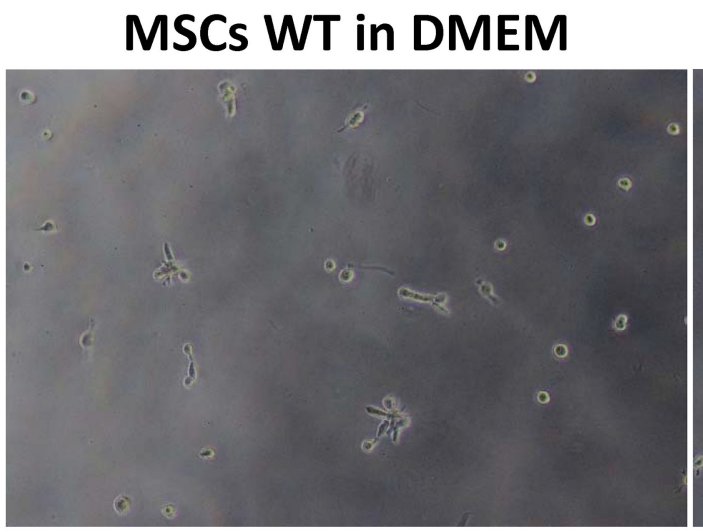

MSCs TNFR2 KO in DMEM

MSCs WT in EGM2

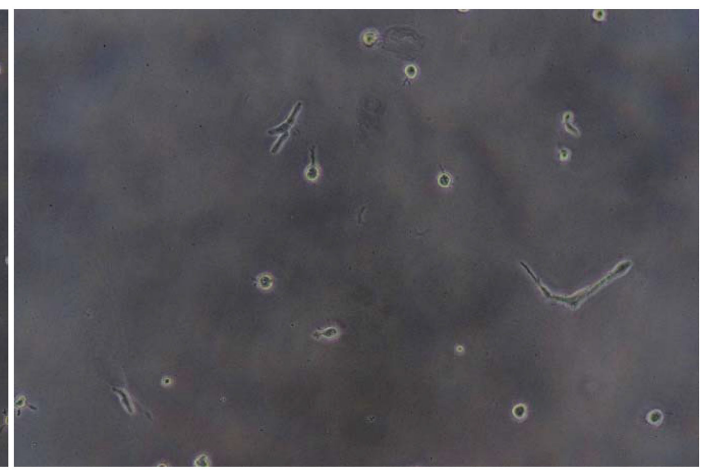

MSCS WT in EGM2

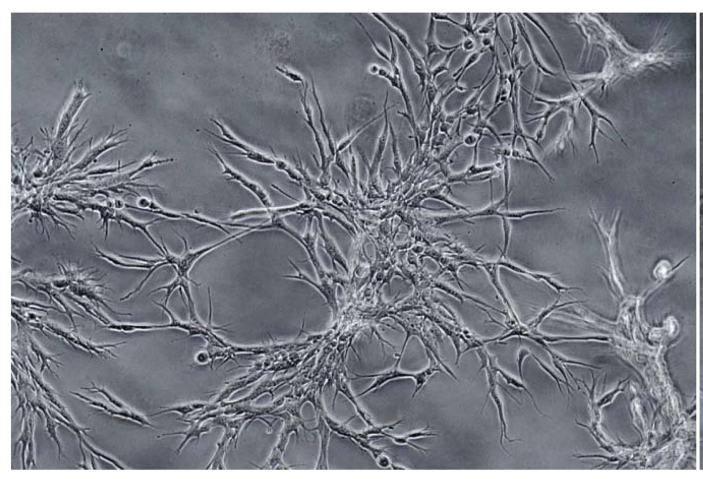

\section{MSCs TNFR2 KO in EGM2}

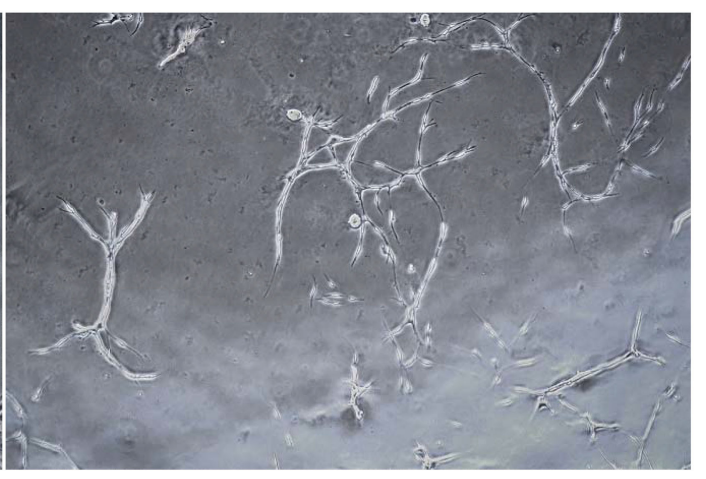

B

C
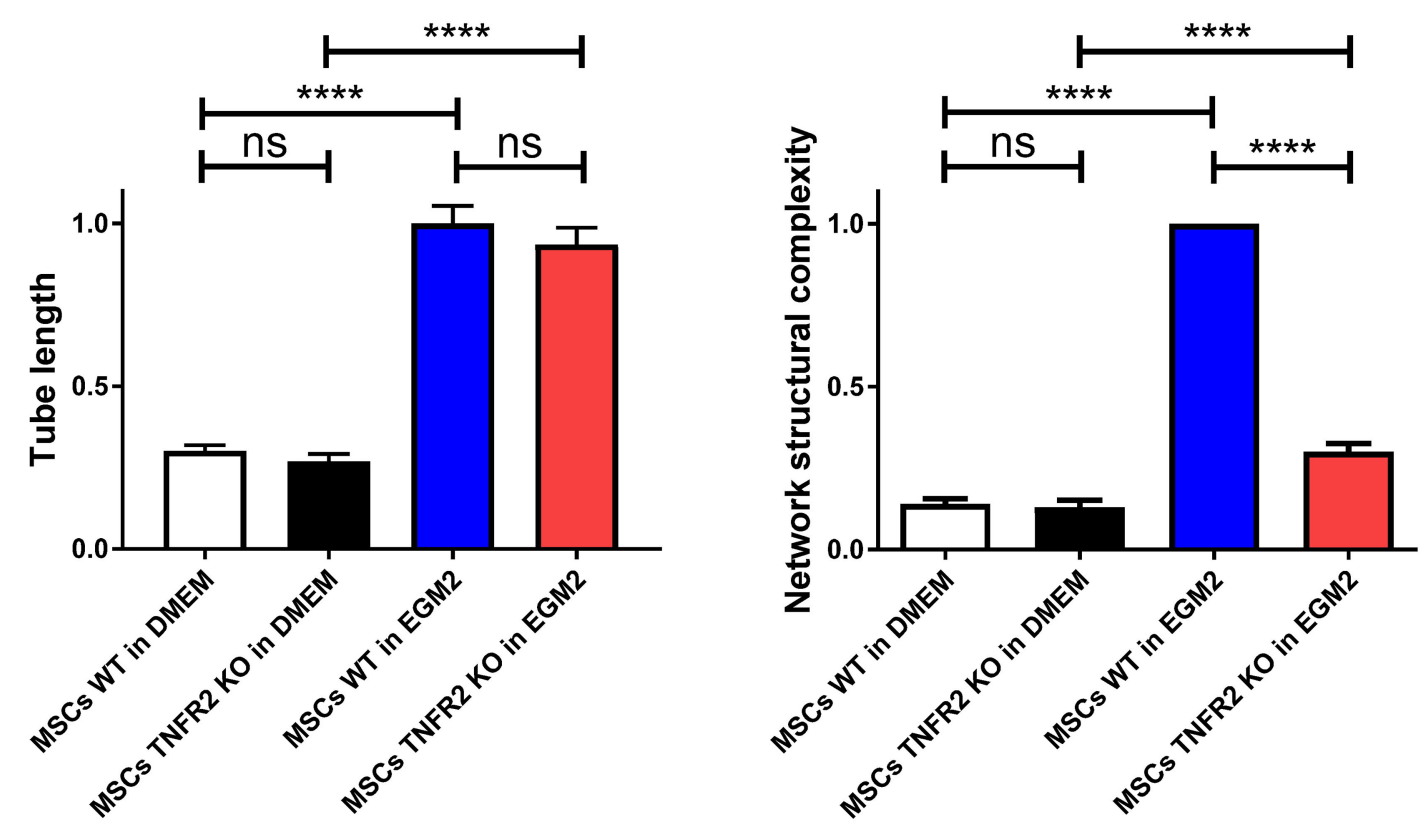

FIGURE 7 | The TNFR2 expression by mesenchymal stem cells (MSCs) is associated with their enhanced tube formation property. To evaluate the involvement of TNFR2 in MSC regenerative feature, wild-type (WT) and TNFR2 knockout (KO)-MSCs (P2 and P3) were cultured on Matrigel using either Dulbecco's modified Eagle's medium (DMEM) standard medium or EGM2 endothelial medium. (A) Pictures were taken every $2 \mathrm{~h}$, using objectives $4 \times$ and $10 \times$ of the inverted microscope in phase-contrast mode. (B) The tube length and (C) network structural complexity of WT and TNFR2 KO-MSCs were further evaluated. Results are collected from three independent experiments $(n=10)$. 
Publisher's Note: All claims expressed in this article are solely those of the authors and do not necessarily represent those of their affiliated organizations, or those of the publisher, the editors and the reviewers. Any product that may be evaluated in this article, or claim that may be made by its manufacturer, is not guaranteed or endorsed by the publisher.
Copyright (๑) 2021 Beldi, Bahiraii, Lezin, Nouri Barkestani, Abdelgawad, Uzan and Naserian. This is an open-access article distributed under the terms of the Creative Commons Attribution License (CC BY). The use, distribution or reproduction in other forums is permitted, provided the original author(s) and the copyright owner(s) are credited and that the original publication in this journal is cited, in accordance with accepted academic practice. No use, distribution or reproduction is permitted which does not comply with these terms. 